\title{
Tradução e adaptação transcultural da versão brasileira do Questionário de Ansiedade Cardíaca
}

\author{
Translation and cross-cultural adaptation of the Brazilian Version of the Cardiac \\ Anxiety Questionnaire
}

\begin{abstract}
Aline Sardinha ${ }^{1}$, Antonio Egidio Nardi' ${ }^{2}$, Georg H. Eifert ${ }^{3}$
${ }^{1}$ Psicóloga. Pesquisadora, Programa de Pós-Graduação em Psiquiatria e Saúde Mental, Laboratório de Pânico e Respiração, Instituto de Psiquiatria, Universidade Federal do Rio de Janeiro (UFRJ), Rio de Janeiro, RJ. ${ }^{2}$ Doutor, livre-docente. Professor associado, Programa de Pós-Graduação em Psiquiatria e Saúde Mental, Laboratório de Pânico e Respiração, Instituto de Psiquiatria, UFRJ. ${ }^{3}$ PhD, Department of Psychology, Chapman University, Orange, CA, EUA.

Este estudo recebeu apoio do Conselho Nacional de Desenvolvimento Científico e Tecnológico (CNPq), processo no ${ }^{\circ} 54411 / 2005-9$.
\end{abstract}

\section{Resumo}

Introdução: É crescente a produção científica brasileira na adaptação de instrumentos internacionais para avaliar ansiedade. A tradução e adaptação transcultural de escalas é um primeiro passo na obtenção de instrumentos válidos que permitam a comparação de diferentes populações. O objetivo do presente estudo foi traduzir e avaliar a equivalência semântica do Cardiac Anxiety Questionnaire, realizando um estudo piloto na população brasileira de diferentes níveis de escolaridade.

Método: O processo de adaptação transcultural envolveu duas traduções e retrotraduções realizadas por avaliadores independentes, avaliação das versões e elaboração de uma versão síntese. Também examinamos os comentários dos participantes sobre a versão preliminar do questionário, os quais foram usados no desenvolvimento da versão final.

Resultados: Para cada item do instrumento, apresentam-se os resultados das quatro etapas. Os participantes com maior grau de escolaridade não apresentaram dificuldades na compreensão do instrumento, tendo apenas apresentado sugestões controversas acerca do item 5. Entretanto, os participantes apenas com escolaridade em nível fundamental relataram dificuldades com os itens 2, 4, 6, 7, 10, 11 e 14. Algumas alterações semânticas foram realizadas com o intuito de facilitar a compreensão do instrumento.

Conclusão: A utilização de duas versões de tradução e retrotradução, discussão sobre a versão síntese e a interlocução com a população-alvo proporcionaram maior segurança ao processo de equivalência semântica da versão final brasileira.

Descritores: Estudos de validação, astenia neurocirculatória e coronariopatia.

\begin{abstract}
Introduction: There has been a growing interest in the cross-cultural application of psychological questionnaires to assess anxiety. The translation and cross-cultural adaptation of the original instrument is the first step in validating an instrument in a new population that will permit comparisons between different populations. The goals of this study were to translate the Cardiac Anxiety Questionnaire, assess its semantic equivalence, and perform a preliminary test with participants from the Brazilian population that were drawn from different educational backgrounds.

Method: The cross-cultural adaptation process consisted of two translations and back translations performed by two independent evaluators; a critical evaluation of the two versions, and the development of a synthesized version. We also examined comments provided by participants on the preliminary version of the questionnaire and used them for the development of the final version.

Results: We report the results of the four stages for each item of the instrument. Participants with tertiary education had no difficulties comprehending the translated items of the questionnaire, only pointing item 5 as ambiguous. Participants from the lower educational level reported comprehension problems regarding items $2,4,6,7,10,11$ and 14 . Some small changes were made in our first version to enhance comprehensibility.
\end{abstract}


Conclusion: The use of two versions of translations, a critical examination of the two versions, and suggestions made by participants resulted in a final Brazilian version with a satisfactory degree of semantic accuracy and semantic equivalence with the original version.

Keywords: Validation studies, neurocirculatory asthenia, coronary disease.

\section{Introdução}

Pacientes cardíacos freqüentemente apresentam comorbidades psiquiátricas ${ }^{1}$. Há muito se identificou a depressão como fator de risco independente para doenças cardiovasculares ${ }^{2,3}$. Mais recentemente, ataques de ansiedade e de pânico também foram relacionados a pior prognóstico em pacientes cardíacos $^{4,5}$. Neste sentido, o reconhecimento de ataques de ansiedade e de pânico em pacientes cardíacos é essencial para evitar complicações sérias ${ }^{6}$.

Furze et al. propuseram que crenças mal adaptadas em relação à doença arterial coronariana podem levar à ansiedade relacionada à saúde, níveis reduzidos de atividade, descondicionamento cardíaco e, portanto, aumento de sintomas e de ansiedade sobre o coração ${ }^{7}$. Tais crenças também são um fator preditivo para abandono da reabilitação cardíaca ${ }^{8}$.

A ansiedade cardíaca é o medo de sensações e estímulos relacionados ao coração e baseados em suas conseqüências negativas percebidas ${ }^{9}$. É um relevante fator promotor de ansiedade porque eventos relacionados ao coração são percebidos como aversivos e perigosos. Demonstrou-se que pessoas que tiveram um ataque cardíaco apresentam crenças mal adaptadas ou mal concebidas que podem ter um efeito deletério sobre a qualidade de vida e funcionamento ${ }^{10}$. Também se observou que os clínicos não obtêm rotineiramente essas crenças mal adaptadas ${ }^{11}$.

Eifert et al. ${ }^{9}$ consideram a ansiedade cardíaca como um tipo de ansiedade específica que parece ser relevante a um número de síndromes clínicas caracterizadas, em parte, por dor torácica e mal-estar psicológico relacionado ao coração. A ansiedade relacionada ao coração pode afetar indivíduos com dor torácica cardíaca e não cardíaca, bem como pacientes com síndrome do pânico. Também pode exacerbar a condição de indivíduos com doença cardíaca conhecida ${ }^{12}$. Infelizmente, a ansiedade cardíaca muitas vezes não é reconhecida como problema primário ou como fator que contribua para pior desfecho de uma condição médica existente, especialmente em pacientes cardiológicos e de emergência.

Instrumentos auto-aplicáveis foram traduzidos e culturalmente adaptados em muitos países, economizando tempo e dinheiro que, do contrário, seriam aplicados em seu desenvolvimento. Ao usar um instrumento que já foi desenvolvido, validado e sistematicamente usado, os pesquisadores poupam recursos valiosos porque o desenvolvimento de um instrumento é uma tarefa que exige grande esforço. Algumas limitações podem ser apontadas nesta abordagem, como o risco de vieses culturais, principalmente se houver diferenças na relevância cultural de um item entre a cultura alvo e a original, que também poderia comprometer futuras comparações transculturais dos dados. Entretanto, mais recentemente, diversos autores optaram por adaptar instrumentos psicométricos internacionais para a população brasileira, ao invés de desenvolver seus próprios instrumentos ${ }^{13-18}$. Essas adaptações, de acordo com demandas psicométricas, podem então ser usadas para comparar os resultados entre as amostras de diferentes países ${ }^{19,20}$.

Um dos processos incluídos na adaptação transcultural é a avaliação da equivalência semântica das escalas ${ }^{21}$. Apesar da falta de consenso em relação aos melhores métodos para conduzi-la, a abordagem mais usada e recomendada inclui a tradução e a retrotradução realizadas por dois tradutores independentes ${ }^{22}$. Neste método, uma pessoa bilíngüe traduz da lingual-fonte para a língua-alvo, e outra pessoa, "cega" ao instrumento original, traduz de volta para a fonte. Posteriormente, são realizadas comparações entre as traduções e retrotraduções para identificar discrepâncias entre a fonte e o alvo, seguidas de discussões com os tradutores bilíngües originais ${ }^{23}$ para identificar possíveis problemas com a equivalência transcultural e atingir uma versão sintética. Isso resulta numa versão preliminarmente adaptada do instrumento, e recomenda-se que ela seja submetida a um processo de validação quantitativa.

o Cardiac Anxiety Questionnaire (CAQ) é um instrumento de 18 itens originalmente desenvolvido por Eifert et al. ${ }^{9}$ para avaliar ansiedade cardíaca. Suas propriedades psicométricas foram avaliadas por processos de análise fatorial e análise de consistência interna e validade convergente e divergente. A estrutura fatorial do questionário inclui três subescalas: medo e preocupação com sensações cardíacas, evitação cardioprotetora de atividades que podem ocasionar sintomas, e atenção e monitoramento cardíaco de estímulos relacionados ao coração. Análises de confiabilidade demonstraram que a consistência interna dos escores total e de subescalas do CAQ foi alta. $\mathrm{O}$ alfa de Cronbach para a escala total foi alto (alfa $=0,83$ ), assim como os coeficientes alfa para cada uma das subescalas (medo, $r=0,83$; evitação, 
$r=0,82$; e atenção, $r=0,69)$. Além disso, a escala apresentou boa validade convergente e divergente, comparada ao Body Sensations Questionnaire $(\mathrm{BSQ})^{24}$, Anxiety Sensitivity Index (ASI) ${ }^{25}$, Activities of Daily Living Scale (ADLS) ${ }^{26}$ e Brief Fear of Negative Evaluation Scale (BFNE) ${ }^{27}$.

O objetivo do presente estudo foi traduzir a avaliar a equivalência semântica do CAQ, realizando um estudo piloto na população brasileira de diferentes níveis de escolaridade. O objetivo foi produzir uma versão preliminarmente adaptada que possa ser subseqüentemente validada. Outro objetivo foi examinar se o CAQ é aplicável a indivíduos de diferentes níveis educacionais.

\section{Método}

O processo de adaptação transcultural do CAQ incluiu quatro passos. Os primeiros três passos se referiam ao processo de tradução: tradução, retrotradução e avaliação de equivalência semântica da versão preliminar em português brasileiro. O próximo passo foi um estudo piloto no qual 23 indivíduos foram solicitados a completar o questionário e indicar se houve qualquer dificuldade ao responder os itens ou sugestões para melhorar o entendimento dos itens. Esta foi uma amostra de conveniência de indivíduos não-clínicos com diferentes níveis de escolaridade, recrutados entre alunos e funcionários do Instituto de Psiquiatria da Universidade Federal do Rio de Janeiro.

No primeiro estágio, duas traduções do instrumento original em inglês para português brasileiro foram independentemente avaliadas por dois psicólogos brasileiros fluentes na língua inglesa. Os tradutores trabalharam de forma independente e não estavam familiarizados com a versão original do questionário, mas tinham conhecimento dos construtos usados no instrumento. Os tradutores foram informados sobre a população-alvo do questionário e foram solicitados a prestar atenção à consistência semântica dos termos e construtos usados, ao invés de apenas traduzir literalmente os itens de inglês para português.

O segundo estágio consistiu da retrotradução para inglês das duas traduções, uma por um psicólogo brasileiro e outra por um psiquiatra brasileiro, também fluente em inglês, independente e cego quanto à escala original.

No estágio três, a avaliação da equivalência semântica foi realizada pelos primeiros dois autores (AS, AE) com o intuito de desenvolver uma versão sintética em português brasileiro baseada nas duas traduções realizadas. O processo de equivalência semântica adotado é parte da adaptação transcultural, com base nas sugestões propostas por Herdman et al. ${ }^{21}$, que foram recentemente aplicadas no Brasil por Reichenheim et al. ${ }^{28}$.

Em princípio, os autores avaliaram a equivalência entre o instrumento original e cada uma das retrotraduções. Subseqüentemente, cada item do instrumento original foi comparado com seu correspondente nas duas versões em português brasileiro. Para compor a versão sintética, alguns itens foram incorporados das duas versões, integralmente ou modificados, ao passo que outros itens surgiram da combinação das duas versões. O resultado desta combinação foi, em algumas vezes, modificado para melhor atender aos critérios de equivalência semântica. No fim deste processo, obtivemos uma versão preliminar em português brasileiro do CAQ que estava pronta para ser testada.

Foi realizado em estudo piloto com a versão sintética em 23 adultos, selecionados de uma amostra de conveniência de universitários e funcionários. Após assinar termo de consentimento informado concordando em participar, os participantes foram solicitados a preencher os questionários e relatar se os itens eram suficientemente claros e se tinham alguma sugestão para melhorar o entendimento do instrumento.

Devido à grande diferença cultural observada na população brasileira, e para garantir o entendimento do questionário por um amplo espectro de pessoas, a amostra foi selecionada com base em nível educacional. Os participantes foram divididos em três categorias: ensino fundamental ( 8 anos de estudo ou menos), ensino médio ( 8 a 11 anos de escolaridade) e curso superior (nível universitário ou maior).

Os participantes não tinham histórico de doenças mentais. Para excluir a possibilidade de um diagnóstico psiquiátrico, os sujeitos foram entrevistados pelos dois primeiros autores usando a Entrevista Clínica Estruturada para o DSM-IV (MINI, versão 5.0) ${ }^{29}$. Todos os participantes foram solicitados a fazer comentários sobre a versão sintética e relatar quaisquer dificuldades que pudessem ter quanto ao entendimento dos itens. Também solicitamos aos participantes que sugerissem palavras ou termos alternativos que pudessem ser entendidos com maior facilidade. Usamos estes comentários e sugestões para desenvolver a versão final do instrumento em português brasileiro.

\section{Resultados}

$\mathrm{O}$ instrumento original, as traduções (T1 e T2), suas respectivas retrotraduções (B1 e B2) e a versão 
sintética (antes das alterações do estudo piloto) podem ser vistos na Tabela 1 . As versões realizadas pelos dois tradutores para os itens $1,3,6,8,9,12$ e 18 foram idênticas, ou muito semelhantes. Em alguns itens, uma versão teve prioridade, ou ambas foram combinadas. Em alguns casos, os autores incluíram ou excluíram termos que foram considerados mais próximos ao significado semântico do item original.

Tabela 1 - Versão original, traduções, retrotraduções e versão sintética do CAQ

\begin{tabular}{llll}
\hline Versão original & Traduções & Retrotraduções & Versão sintética \\
\hline 1. I pay attention to my heart & T1. Presto atenção nas & B1. I pay attention to the & 1. Presto atenção às batidas \\
beat. & batidas do meu coração. & beating of my heart. & do meu coração. \\
& T2. Eu presto atenção aos & B2. I am aware of my &
\end{tabular}

2. I avoid physical exertion. T1. Evito atividades físicas extenuantes.

T2. Eu evito esforço físico.

3. My racing heart wakes me up at night.

\section{Chest pain/discomfort} wakes me up at night.

5. I take it easy as much as possible.

6. I check my pulse.

7. I avoid exercise or other physical work.

\section{I can feel my heart in my chest.}

T1. Meu coração palpitante me acorda à noite.

T2. Meu coração disparado me acorda à noite.

\section{T1. Dores no coração ou} desconforto me acordam a noite.

T2. Dor/desconforto no peito me acorda à noite.

T1. Tento não me estressar sempre que possível.

$\mathrm{T} 2$. Eu pego leve sempre que possível

heartbeats.

B1. I avoid exhausting physical activities.

B2. I avoid exertion (or physical strain).

B1. My beating heart wakes me up at night.

B2. My fast heartbeat wakes me at night.

B1. Pain chest or discomfort wake me up at night.

B2. Chest pain/discomfort

awakes me at night.

B1. I try not to stress myself when is possible.

2. Evito esforço físico.

T1. Verifico minha pulsação.

T2. Eu verifico minha pulsação

T1. Evito fazer exercício ou outras atividades físicas.

T2. Eu evito me exercitar ou fazer outro tipo de trabalho físico.

\section{T1. Consigo sentir meu} coração dentro do meu peito. T2. Eu consigo sentir meu coração no meu peito.

9. I avoid activities that make T1. Evito atividades que my heart beat faster. façam meu coração bater mais rapidamente.

T2. Eu evito atividades que façam meu coração bater mais rápido.

10. If tests come out normal, T1. Se os exames apresentam I still worry about my heart. resultados normais, continuo me preocupando com o meu coração.

T2. Mesmo se os exames se mostrarem normais, eu me preocupo com o meu coração.
B2. I take it easy whenever possible.

B1. I check my pulse.

B2. I check my pulse.

B1. I avoid doing exercise or physical activity.

B2. I avoid exercising and other kinds of physical activities.

B1. I can feel my heart inside 8 . Posso sentir meu coração my chest. no meu peito.

B2. I can feel my heart inside my chest.

B1. I avoid activities that make my heart beat faster. B2. I avoid activities which

9. Evito atividades que acelerem meu coração.
B1. If the results of the exams are normal, I continue to worry about my heart. B2. Even when my medical exams are normal I worry about my heart.
10. Mesmo que os meus exames estejam normais, eu continuo me preocupando com meu coração.
3. Meu coração acelerado me acorda à noite.

4. Dor ou desconforto no peito me acordam à noite.

5. Tento não me estressar o máximo possível.

6. Verifico minha pulsação.

7. Evito fazer exercício ou outras atividades físicas. 


\begin{tabular}{|c|c|}
\hline Versão original & Traduções \\
\hline $\begin{array}{l}\text { 11. I feel safe being around a } \\
\text { hospital, physician or other } \\
\text { medical facility. }\end{array}$ & $\begin{array}{l}\text { T1. Me sinto seguro estando } \\
\text { perto de um hospital, médico } \\
\text { ou instalação hospitalar. } \\
\text { T2. Eu me sinto seguro(a) } \\
\text { estando próximo(a) a um } \\
\text { hospital, a um médico ou a } \\
\text { outro serviço de saúde. }\end{array}$ \\
\hline $\begin{array}{l}\text { 12. I avoid activities that } \\
\text { make me sweat. }\end{array}$ & $\begin{array}{l}\text { T1. Evito atividades que me } \\
\text { façam suar. } \\
\text { T2. Eu evito atividades que } \\
\text { me façam suar. }\end{array}$ \\
\hline
\end{tabular}

13. I worry that doctors do T1. Me preocupo em que os not believe my symptoms are médicos não acreditem que real.

When I have chest discomfort or when my heart is beating fast:

meus sintomas são

verdadeiros

T2. Eu me preocupo com a possibilidade de os médicos não acreditarem que os meus sintomas são reais.

T1. Quando sinto desconforto no peito ou quando meu coração bate rápido:
T2. Quando eu tenho um desconforto no peito ou quando o meu coração está batendo rápido:

14. I worry that I may have a T1. Me preocupo em ter um heart attack.

\section{I have difficulty} concentrating on anything else.

\section{I get frightened.}

17. I like to be checked out by a doctor.
18. I tell my family or friends.

T2. Eu me preocupo se eu terei um ataque cardíaco.

T1. Tenho dificuldade em me concentrar em qualquer outra coisa. me concentrar em alguma coisa.

T1. Quero ser examinado por um médico. 2. Eu gosto de ser examinado(a) por um médico.

T1. Conto a situação para minha família ou amigos.

\section{Retrotraduções \\ Versão sintética}

B1. I feel safe near a hospital, 11. Sinto-me seguro(a) doctor or hospital installation.

B2. I feel safe when I am close to a hospital, a doctor or health service.

B1. I avoid activities that make me sweat.

B2. I avoid activities which make me sweat.

B1. I worry that the doctors do not believe that my symptoms are real.

B2. I worry about the possibility of doctors not believing my symptoms are real.

B1. When I feel a discomfort Quando eu tenho desconforto in the chest or when my heart no peito ou quando meu beats fast: coração está acelerado:

B2. When I feel chest discomfort or my heartbeats are fast:

B1. I worry about a heart attack.

B2. I worry about having a heart attack.

B1. It is difficult for me to focus in other things.

B2. I find it hard to T2. Eu tenho dificuldade em concentrate in something.
T1. Fico amedrontado.
T2. Eu fico assustado(a).

\section{B1. I feel frightened.}
B2. I get scared.

14. Preocupa-me que posso ter um ataque cardíaco.

15. Tenho dificuldade em me concentrar em qualquer outra coisa.

13. Preocupa-me que os médicos não acreditem que meus sintomas sejam verdadeiros.

B1. I want to be examined by a doctor. B2. I like being examined by a doctor. T2. Eu conto para a minha família ou amigos
B1. I tell the situation to my friends or family. B2. I tell my family and friends about it.
18. Conto para minha família ou amigos. 
No item 2, a T2 "Evito esforço físico" foi escolhida porque "extenuantes" não é uma palavra geralmente usada no Brasil. Portanto, a palavra "esforço", em inglês "effort", foi escolhida para transmitir a idéia de esforço físico. Também no item 4, a T2 foi escolhida, pois apresenta tanto "dor" quanto "desconforto" relacionado à palavra "peito", ao passo que, na $\mathrm{T} 1$, a estrutura gramatical da sentença pode tornar o item mais ambíguo.

O item 5 foi o mais controverso, uma vez que a expressão "take it easy" não existe em português brasileiro e pode ser traduzida de formas diferentes, dependendo da situação. Apesar da T2 "Eu pego leve sempre que possível" ter atingido a retrotradução mais literal "I take it easy whenever possible", optamos pela T1 "Tento não me estressar sempre que possível", pois pareceu mais apropriada por se referir à evitação de situações estressantes. Também neste item, a expressão "as much as possible" foi traduzida pelos dois tradutores por "sempre que possível", que literalmente significa "whenever possible". Entretanto, "sempre que possível" e "o máximo possível", que seriam a tradução literal para a expressão, têm significados intercambiáveis em português, e ambos podem ser usados nesta situação.

No item 7, a T1 foi escolhida. Embora a expressão "physical work" pudesse ser literalmente traduzida por "trabalho físico", esta não é uma expressão usada em português brasileiro, e "atividade física" seria mais apropriada para se referir a essas situações. O item 10 resultou de uma combinação das duas traduções. A palavra "se" foi corretamente traduzida em T1 por "se", mas a adaptação realizada por T2, usando "mesmo que" pareceu melhor, uma vez que não é comum, embora correto, usar "se" no Brasil para expressar este tipo de idéia.

$\mathrm{O}$ item 11 é uma adaptação da $\mathrm{T} 2$ "Eu me sinto seguro(a) estando próximo(a) a um hospital, a um médico ou a outro serviço de saúde". As alterações feitas resultaram de observações gramaticais de que não é correto, na linguagem escrita, começar uma sentença com o pronome "me", mesmo que seja comumente realizado na língua falada. Além disso, a exclusão do artigo "a" e o uso do plural das palavras "hospital", "doctor" e "medical facility" dariam um sentido mais geral em português brasileiro. Isso acontece porque o uso do artigo significa que a pessoa pode estar se referindo a um médico específico. Desta forma, a forma final deste item foi: "Sinto-me seguro(a) estando próximo a hospitais, médicos ou outros serviços de saúde". No item 13, a T1 foi escolhida, mas novamente tivemos que corrigir o pronome que começava a sentença.

Os itens 14,15 e 16 pertenciam à segunda seção do instrumento, referindo-se a "When I have chest discomfort or when my heart is beating fast", que foi traduzido por "Quando eu tenho desconforto no peito ou quando meu coração está acelerado".

No item 14, as duas traduções não retratavam o senso de probabilidade do termo "may", portanto nenhuma retrotradução apresentou essa idéia. Neste caso, os autores decidiram incluir o verbo "posso" para expressá-la. No final, a versão traduzida preliminar resultou em "Preocupa-me que posso ter um ataque cardíaco". Para o item 15, usamos a T2.

Para o item 16, a tradução exata seria a feita pelo primeiro tradutor: "Eu fico amedrontado". Entretanto, a palavra "amedontrado" poderia não ser familiar a algumas partes da população brasileira. Ela é muitas vezes substituída pela expressão "com medo", que é mais comum em português brasileiro escrito e oral. Neste caso, os autores decidiram aceitar a sugestão do segundo tradutor, alterando-a para "Fico com medo".

Finalmente, no enunciado 17, a T2 foi escolhida. Em todos os casos, o pronome "I", que seria traduzido por "Eu", foi excluído do início das sentenças em todos os itens, pois é mais comum suprimir o pronome inicial do início de sentenças em português brasileiro, já que a conjugação do verbo indica a pessoa do sujeito.

Durante o estudo piloto do questionário com os sujeitos, nosso propósito foi testar o entendimento e ocasionalmente modificar qualquer termo ou sentença que pudesse ser de difícil compreensão. Os autores buscaram examinar a possibilidade de usar o questionário em um amplo espectro de indivíduos oriundos de diferentes níveis culturais e educacionais.

A amostra usada no estudo piloto foi composta de seis homens e 17 mulheres, com idades variando de 20 a 75 anos. Estatística descritiva e freqüências desta amostra estão apresentadas na Tabela 2. 
Tabela 2 - Estatística descritiva da amostra do estudo piloto

\begin{tabular}{|c|c|c|c|c|}
\hline & Fundamental & Médio & Superior & Total \\
\hline \multicolumn{5}{|l|}{ Gênero } \\
\hline Masculino & 0 & 0 & 6 & 6 \\
\hline Feminino & 7 & 6 & 4 & 17 \\
\hline Total & 7 & 6 & 10 & 23 \\
\hline \multicolumn{5}{|l|}{ Idade } \\
\hline Mínima & 47 & 20 & 24 & 20 \\
\hline Máxima & 70 & 75 & 51 & 75 \\
\hline Média & 55,57 & 38,83 & 38,10 & 43,61 \\
\hline Desvio padrão & 8,18 & 19,94 & 11,75 & 15,19 \\
\hline
\end{tabular}

A maioria das sugestões derivou do grupo com nível universitário, principalmente em relação à semelhança semântica de alguns enunciados, que pareceu um tanto repetitiva. Esta observação foi realizada com menos freqüência nos outros grupos. Entretanto, os itens apontados como semelhantes foram aqueles que pertenciam às mesmas subescalas, e logicamente era esperado que se referissem aos mesmos assuntos. Apesar dessas observações, decidimos manter a estrutura original do instrumento para manter ao máximo suas propriedades psicométricas, pois o objetivo deste estudo foi traduzi-lo e adaptá-lo culturalmente, fazendo o mínimo de alterações estruturais para preservar a validade semântica. Além disso, outra expectativa foi que esses sujeitos poderiam oferecer sugestões valiosas para melhorar o instrumento. Essas expectativas foram atingidas, e a maioria das alterações sugeridas por esses participantes foi de alguma utilidade.

Além de questionar os sujeitos sobre seu entendimento da escala e solicitar sugestões, o autor pediu que os sujeitos respondessem aos itens e, assim, preenchessem o questionário. Isso serviu para lidar com um potencial viés de desejo social, pois pensamos que alguns sujeitos, principalmente aqueles com menos anos de escolaridade, poderiam se sentir envergonhados em admitir que não entenderam algum item e apenas declarar que a escala era compreensível. Embora essas medidas fossem feitas, somente quatro sujeitos do grupo de escolaridade mais baixa $(n=7)$ declararam que tinham algum problema no entendimento dos enunciados. Além do mais, nenhum deles voluntariamente apresentou alguma sugestão de alteração. Eles simplesmente não responderam ao item e, quando questionados pelos pesquisadores, declararam que não entenderam exatamente o que o item significava. Os itens citados pelos participantes deste grupo foram os itens $2,4,6$, $7,10,11$ e 14 . Dois deles também tiveram dificuldade em perceber que os últimos cinco itens eram relativos à situação descrita como "Quando eu tenho desconforto no peito ou quando meu coração está acelerado".

Nenhum dos participantes com 8 a 11 anos de escolaridade relatou quaisquer problemas referentes ao entendimento do instrumento e, portanto, não sugeriram nenhuma alteração.

No grupo de ensino superior, todos os participantes declararam ter entendido completamente os itens. Por outro lado, esses sujeitos apresentaram diversas sugestões e fizeram alterações relevantes, algumas das quais foram escolhidas pelos autores na versão final da escala. Novamente neste grupo, houve reclamação sobre os últimos cinco itens relativos à mesma situação, e alguns participantes relataram ter de ler o enunciado "Quando eu tenho desconforto no peito ou quando meu coração está acelerado" mais de uma vez para entender que todos os cinco itens eram relacionados ao mesmo enunciado introdutório.

Dos participantes com ensino superior que apresentaram sugestões, todos mencionaram que, nos itens 2, 7 e 12, deveria ser especificado que a evitação das atividades era devida à ansiedade relacionada ao coração. Alguns exemplos dados por esses sujeitos foram que eles podem evitar esforço físico (item 2) porque são preguiçosos, e não porque têm medo dos sintomas cardíacos, ou, no item 12, podem evitar atividades que os fazem suar porque não querem ter que tomar banho mais do que uma vez por dia, ou porque não querem estragar o penteado. Embora esses enunciados tivessem algum sentido, decidimos não alterar os itens ainda mais, pois alterações dessa magnitude poderiam alterar as propriedades psicométricas do instrumento original. 
As controvérsias relativas ao item 5 , encontradas no processo de tradução, foram novamente verificadas neste passo. Os participantes concordaram que a palavra "tento", do início do enunciado, é diferente de realmente conseguir não se estressar. Assim, ficaram confundidos na resposta, pois normalmente tentam não se estressar, mas raramente o conseguem. Além disso, alguns deles mencionaram que o significado da palavra "estressar" é muito amplo e pode se referir a estresse físico ou psicológico. Neste caso, alguém poderia evitar situações que pudessem causar estresse psicológico, mas se sentir confortável em se engajar em atividades físicas estressantes. Este item foi trocado na versão final, na qual os autores optaram por manter a tradução mais literal sugerida pelo segundo tradutor: "Eu pego leve sempre que possível".

Finalmente, dois participantes do grupo de maior nível de escolaridade sugeriram que, no item 10, a palavra "resultados" fosse adicionada a "exames" para dar a idéia de "resultados de exames", ao invés de apenas "exames". Consideramos esta adição irrelevante para a compreensão geral do item e, portanto, não fizemos nenhuma alteração.

Outro participante sugeriu que, no item 18, o enunciado "Conto para minha família ou amigos" daria a impressão de que a pessoa está com vontade de contar para um grande número de pessoas sobre seus sintomas, porque a palavra "família" é um substantivo coletivo e a palavra "amigos" está no plural. Como sugerido, a palavra "Conto" foi substituída por "Falo", que seria mais adequada neste caso.

Também incorporamos algumas das sugestões feitas pelos sujeitos na aplicação do estudo piloto do questionário na versão final. Embora a maioria dos comentários fosse interessante, alguns não puderam ser incorporados sem alterar a estrutura psicométrica, e outros representariam mudanças irrelevantes em termos de significado. Com relação aos problemas apontados nos itens 14 a 18 , que se referiam à mesma situação, decidiu-se que o formato da folha do questionário deveria ser modificado para deixar mais explícito que esses itens se referem ao mesmo enunciado.

\section{Discussão}

Conceitos como ansiedade, estresse e doença têm significados distintos e podem estar associados a diferentes comportamentos para pessoas que vivem em contextos sociais diferentes. Portanto, antes de aplicar questionários psicológicos desenvolvidos para um determinado contexto cultural em outro contexto, é necessário conduzir uma avaliação meticulosa da equivalência semântica entre um instrumento original e sua versão adaptada. De acordo com as diretrizes propostas na literatura ${ }^{30}$, esta adaptação transcultural deveria enfatizar a equivalência semântica, ao invés da tradução literal dos termos. O objetivo é expressar conceitos de maneira que façam sentido à nova população-alvo. Esta é a abordagem geral adotada neste estudo. Por outro lado, sempre que foi possível no processo de tradução, escolhemos uma tradução mais próxima ao original para manter o significado central sem comprometer uma compreensão precisa do enunciado e preservar as propriedades psicométricas do questionário.

Embora seja, de certa forma, controverso se este é o melhor método de avaliar a equivalência semântica, o presente estudo adotou este processo através de duas traduções e duas retrotraduções. O uso de dois tradutores foi considerado útil, pois as duas versões puderam ser comparadas e discutidas com o intuito de desenvolver uma versão sintética. Este procedimento permitiu uma discussão extensa quando foram identificadas discrepâncias para chegar à melhor solução possível. Embora os tradutores não tivessem conhecimento do questionário e trabalhassem de forma independente, sua experiência na área e a familiaridade com os construtos foi essencial e útil no processo de determinação de equivalência semântica. Alguns problemas geralmente encontrados na tradução de instrumentos da língua original para outra resultam da falta de familiaridade dos tradutores com a área de pesquisa. Os tradutores especialistas, neste caso, lançaram luz sobre questões que poderíamos ter falhado em identificar, uma vez que os autores estão tipicamente muito próximos do assunto e certamente não estão cegos quanto ao estudo.

Uma limitação deste estudo foi o pequeno número de sujeitos $(\mathrm{n}=23)$ na aplicação do estudo piloto para avaliação do instrumento. Essa limitação foi, de certa forma, atenuada pelo uso de pessoas de diferentes níveis de escolaridade. Além disso, o uso de sujeitos com diferentes níveis de escolaridade nos garantiu a possibilidade de avaliar a viabilidade da aplicação do instrumento na população brasileira, que é claramente composta de indivíduos com níveis de escolaridade que variam enormemente. Quanto a isso, é importante destacar que algumas pessoas com menos de 8 anos de escolaridade relataram problemas na compreensão de alguns itens. Esses dados lançam luz sobre as questões que podem ser encontradas ao usar este instrumento nesta população. Embora deva ser esclarecido por estudos futuros de validação desta versão do questionário, no presente momento, os 
dados derivados do uso da versão brasileira do CAQ em indivíduos com menos de 8 anos de escolaridade devem ser cuidadosamente examinados, tendo essas limitações em mente.

A aplicação do estudo piloto gerou sugestões úteis, sendo que algumas foram incorporadas na versão final do instrumento. Também foi aparente que a maioria dos participantes não teve problemas em entender as situações descritas no questionário e, em muitas ocasiões, conseguiram identificar a si próprios agindo ou sentindo algo semelhante aos enunciados relativos a medo, evitação e preocupação com sintomas cardíacos. Parece razoável concluir que este breve questionário pode ser usado para identificar ansiedade cardíaca comum que possa estar presente em sujeitos com ou sem doenças cardiovasculares, assim como em outros países ${ }^{12}$.

Embora seja um importante passo no processo de validação de um questionário, a adaptação transcultural e a equivalência semântica são apenas o primeiro passo. Além disso, o instrumento também deve demonstrar boa confiabilidade e validade. Por exemplo, o questionário deve ser sensível e apresentar suficiente especificidade para ser útil para propósitos de diagnóstico diferencial ${ }^{31}$. Este estudo é o primeiro passo para determinar esses fatores para a versão em português do CAQ e sua aplicabilidade na população brasileira. A versão final em português brasileiro do CAQ (ver Anexo 1) agora deverá ser submetida a uma análise de sua estrutura fatorial e consistência interna. A validade convergente e divergente também precisará ser avaliada em futuros estudos.

\section{Agradecimentos}

Os autores gostariam de agradecer à Adriana Nunan, Gisele Dias, Maria Cristina Ferreira, Michele Levitan e Rafael Freire por suas grandes contribuições ao processo de tradução, retrotradução e adaptação.

\section{Referências}

1. Bankier B, Januzzi JL, Littman AB. The high prevalence of multiple psychiatric disorders in stable outpatients with coronary heart disease. Psychosom Med. 2004;66(5):645-50.

2. Frasure-Smith N, Lesperance F, Talajic M. Depression following myocardial infarction. Impact on 6-month survival. JAMA. 1993;270(15):1819-25.

3. Frasure-Smith N, Lesperance F, Talajic M. Depression and 18month prognosis after myocardial infarction. Circulation. 1995;91(4):999-1005.

4. Fleet R, Lesperance F, Arsenault A, Grégoire J, Lavoie K, Laurin $\mathrm{C}$, et al. Myocardial perfusion study of panic attacks in patients with coronary artery disease. Am J Cardiol. 2005;96(8):1064-8.
5. Shioiri T, Kojima M, Hosoki T, Kitamura H, Tanaka A, Bando T, et al. Momentary changes in the cardiovascular autonomic system during mental loading in patients with panic disorder: a new physiological index "rho(max)". J Affect Disord. 2004;82(3):395401.

6. Katerndahl D. Panic plaques: panic disorder \& coronary artery disease in patients with chest pain. J Am Board Fam Pract. 2004;17(2):114-26.

7. Furze G, Lewin RJ, Murberg T, Bull P, Thompson DR. Does it matter what patients think? The relationship between changes in patients' beliefs about angina and their psychological and functional status. J Psychosom Res. 2005;59(5):323-9.

8. Yohannes AM, Yalfani A, Doherty P, Bundy C. Predictors of drop-out from an outpatient cardiac rehabilitation programme. Clin Rehabil. 2007;21(3):222-9.

9. Eifert GH, Thompson RN, Zvolensky MJ, Edwards K, Frazer NL, Haddad JW, et al. The cardiac anxiety questionnaire: development and preliminary validity. Behav Res Ther. 2000;38(10):1039-53.

10. Jiang W, Kuchibhatla M, Cuffe MS, Christopher EJ, Alexander JD, Clary GL, et al. Prognostic value of anxiety and depression in patients with chronic heart failure. Circulation. 2004;110(22):3452-6.

11. Furze G, Bull P, Lewin, RJ, Thompson DR. Development of the York Angina Beliefs Questionnaire. J Health Psychol. 2003;8(3):307-15.

12. Hoyer J, Eifert GH, Einsle F, Zimmermann K, Krauss S, Knaut M, et al. Heart-focused anxiety before and after cardiac surgery. J Psychosom Res. 2008;64(3):291-7.

13. Duarte PS, Miyazaki MCOS, Ciconelli RM, Sesso R. Tradução e adaptação cultural do instrumento de avaliação de qualidade de vida para pacientes renais crônicos (Kdqol-Sftm). Rev Assoc Med Bras. 2003;49(4):375-81.

14. Camargo IB, Contel JOB. Tradução e adaptação de questionários norte-americanos para a avaliação de habilidades e conhecimentos na prática psiquiátrica brasileira. R Psiquiatr RS. 2004;26(3):28899.

15. Mattos P, Segenreich D, Saboya E, Louzã M, Dias G, Romano M. Adaptação transcultural para o português da escala Adult SelfReport Scale para avaliação do transtorno de déficit de atenção/ hiperatividade (TDAH) em adultos. Rev Psiq Clin. 2006;33(4):188-94

16. Souza FP, Foa EB, Meyer E, Niederauer KG, Raffin AL, Cordioli AV. Obsessive-Compulsive Inventory and Obsessive-Compulsive Inventory-Revised scales: translation into Brazilian Portuguese and cross-cultural adaptation. Rev Bras Psiquiatr. 2008;30(1):42-6.

17. Goldfeld PRM, Wiethaeuper D, Terra L, Baumgardt R, Lauermann M, Mardini V, et al. Adaptação transcultural do Inventory of Countertransference Behavior (ICB) para o português brasileiro. Rev Psiquiatr RS. 2007;29(1):56-62.

18. Levitan MN, Nascimento I, Mezzasalma MA, Freire RC, Nardi AE. Semantic equivalence of the Brazilian Portuguese Version of the Social Avoidance and Distress Scale (SADS). Rev Psiq RS. 2008. In press.

19. Mas Pons R, Escriba Agüir V. La versión castellana de la escala The Nursing Stress Scale. Proceso de adaptación transcultural. Rev Esp Salud Publica. 1998;72(6):529-38.

20. Yu DS, Lee DT, Woo J. Issues and challenges of instrument translation. West J Nurs Res. 2004;26(3):307-20.

21. Herdman M, Fox-Rushby J, Badia X. "Equivalence" and the translation and adaptation of health-related quality of life questionnaires. Qual Life Res. 1997;6(3):237-47.

22. Brislin RW. Back-translation for cross-cultural research. J Cross Cult Psychol. 1970;1(3):185-216.

23. Weeks A, Swerissen H, Belfrage J. Issues, challenges, and solutions in translating study instruments. Eval Rev. 2007;31(2);153-65.

24. Chambless DM, Caputo GC, Bright P, Gallagher R. Assessment of fear in agoraphobics: the body sensations questionnaire and agoraphobia cognitions questionnaire. J Cons Clin Psychol. 1984;52(6):1090-7.

25. Peterson RA, Reiss S. Anxiety sensitivity index manual. 2nd ed. Worthington: International Diagnostic Systems; 1992. 
26. Linton SJ. Activities of daily living scale for patients with chronic pain. Percept Mot Skills. 1990;71(3 Pt 1):722.

27. Leary MR. A brief version of the Fear of Negative Evaluation scale. Person Soc Psychol Bull. 1993;9:371-5.

28. Reichenheim ME, Moraes CL. Operacionalização de adaptação transcultural de instrumentos de aferição usados em epidemiologia. Rev Saude Publica. 2007;41(4):665-73.

29. Sheehan DV, Lecrubier Y, Sheehan KH, Amorim P, Janavs J, Weiller E, et al. The Mini International Neuropsychiatric Interview (MINI): the development and validation of a structured diagnostic psychiatric interview for DSM-IV and ICD-10. J Clin Psychiatry. 1998;59 Suppl. 20:22-33.

30. Vilete L, Figueira I, Coutinho E. Adaptação transcultural para o português do Social Phobia Inventory (SPIN) para utilização entre estudantes adolescentes. Rev Psiquiatr RS. 2006;28(1):40-8.

31. Berger W, Mendlowicz MV, Souza WF, Figueira I. Equivalência semântica da versão em português da Post-Traumatic Stress Disorder Checklist-Civilian Version (PCL-C) para rastreamento do transtorno de estresse pós-traumático. Rev Psiquiatr RS. 2004;26(2):167-75. 


\section{Anexo 1 - Questionário de Ansiedade Cardíaca (versão adaptada)}

\section{Questionário de Ansiedade Cardíaca}

Conto para minha família ou amigos

Por favor, avalie cada item marcando a resposta que melhor corresponde a você:

\begin{tabular}{|c|c|c|c|c|c|}
\hline & Nunca & Raramente & Às vezes & Freqüentemente & Sempre \\
\hline \multicolumn{6}{|l|}{ Presto atenção nas batidas do meu coração } \\
\hline \multicolumn{6}{|l|}{ Evito esforço físico } \\
\hline \multicolumn{6}{|l|}{ Meu coração acelerado me acorda à noite } \\
\hline \multicolumn{6}{|l|}{$\begin{array}{l}\text { Dor ou desconforto no peito me acordam à } \\
\text { noite }\end{array}$} \\
\hline \multicolumn{6}{|l|}{ Pego leve o máximo possível } \\
\hline \multicolumn{6}{|l|}{ Verifico minha pulsação } \\
\hline \multicolumn{6}{|l|}{$\begin{array}{l}\text { Evito fazer exercícios ou outras atividades } \\
\text { físicas }\end{array}$} \\
\hline \multicolumn{6}{|l|}{ Posso sentir meu coração no meu peito } \\
\hline \multicolumn{6}{|l|}{ Evito atividades que acelerem meu coração } \\
\hline \multicolumn{6}{|l|}{$\begin{array}{l}\text { Mesmo que os exames estejam normais, eu } \\
\text { continuo me preocupando com meu coração }\end{array}$} \\
\hline \multicolumn{6}{|l|}{$\begin{array}{l}\text { Sinto-me seguro estando próximo a } \\
\text { hospitais, médicos ou outros serviços de } \\
\text { saúde }\end{array}$} \\
\hline \multicolumn{6}{|l|}{ Evito atividades que me façam suar } \\
\hline $\begin{array}{l}\text { Preocupa-me que os médicos não acreditem } \\
\text { que meus sintomas sejam verdadeiros }\end{array}$ & & & & & \\
\hline
\end{tabular}

Quando eu tenho desconforto no peito ou quando meu coração está acelerado:

\begin{tabular}{|l|l|l|l|l|l|}
\hline & Nunca & Raramente & Às vezes & Freqüentemente & Sempre \\
\hline $\begin{array}{l}\text { Preocupa-me que posso ter um ataque } \\
\text { cardíaco }\end{array}$ & & & & & \\
\hline $\begin{array}{l}\text { Tenho dificuldade em me concentrar em } \\
\text { qualquer outra coisa }\end{array}$ & & & & & \\
\hline Fico com medo & & & & & \\
\hline Gosto de ser examinado por um médico & & & & & \\
\hline Conto para minha família ou amigos & & & & & \\
\hline
\end{tabular}

